\title{
Oxygen vacancy, oxygen vacancy-vacancy pairs and Frenkel defects in cubic lutetium oxide
}

\author{
A. Shyichuk*, E. Zych \\ Faculty of Chemistry, University of Wrocław, 14 F. Joliot-Curie, 50-383 Wrocław, Poland \\ * andrii.shyichuk@chem.uni.wroc.pl, +48 713757265 \\ eugeniusz.zych@chem.uni.wroc.pl, +48 713757248 \\ * corresponding author:
}

\section{Supplementary information}

\section{Geometry optimization}

Quantum Espresso 6.1 (https://www.quantum-espresso.org/) module pw.x (total energy calculations and geometry optimization) was used with mostly default settings. Perdew-Zunger local density approximation (LDA) functional (10.1103/PhysRevB.23.5048) was used. Crucial settings are shown in Table S1.

Table S1. Selected settings used in Quantum Espresso calculations.

\begin{tabular}{cc}
\hline \multicolumn{2}{c}{ Section \&CONTROL } \\
\hline calculation & 'vc-relax' \\
etot_conv_thr & $1 \mathrm{E}-5$ \\
forc_conv_thr & 0.0001 \\
nstep & 700 \\
& \\
\hline \multicolumn{2}{c}{ Section \&SYSTEM } \\
ecutwfc & 40 / 50 \\
ecutrho & $400 / 500$ \\
occupations & 'smearing' \\
smearing & 'gaussian' \\
degauss & 0.001 \\
nspin & 1 or $2 *$ \\
tot_charge & 2 / 1 / 0 / - 1 / - 2 *
\end{tabular}

\begin{tabular}{cc}
\hline \multicolumn{2}{c}{ Section \&ELECTRONS } \\
\hline electron_maxstep & 200 \\
conv_thr & $1.0 \mathrm{D}-7$ \\
diago_thr_init & $1.0 \mathrm{E}-4$ \\
startingpot & 'atomic' \\
mixing_mode & 'plain' \\
mixing_beta & 0.5 \\
mixing_ndim & 8 \\
diagonalization & 'david' \\
\hline \multicolumn{2}{c}{ Section \&IONS } \\
\hline ion_dynamics & 'bfgs' \\
\hline cell_dynamics & 'bfgs'
\end{tabular}

* tot_charge is total charge on the system, while nspin controls spin polarization (1 for unpolarized and 2 for polarized); spinpolarization was used for systems with odd tot_charge.

All of the systems used ecutwfc $=40$ and ecutrho $=400$, with two exceptions, and $\mathrm{Lu}_{2} \mathrm{O}_{3}: V_{\mathrm{O} 33}$, which used ecutwfc $=50$ and ecutrho $=500$. The exception was made due to problematic convergence with the initially used values $(40,400)$.

\section{Pseudopotentials}

The pseudopotentials (PPs) used were ultrasoft (USPP), generated by the Vanderbilt code version 7.3.6. The oxygen PP recipe (PP generation input file) from GBRV group (10.1016/j.commatsci.2013.08.053), version 1.2 was used. The Lu USPP recipe was kindly provided by co-author of the GBRV set, Kevin F. Garrity. The potential had the $4 \mathrm{f}$ electrons in core; the valence electrons were effectively of a $d$-element, not an $f$-element. 


\section{Initial geometry of $\mathrm{Lu}_{2} \mathrm{O}_{3}$ cell}

Initial geometry (in from of fractional coordinates of atoms) was generated by Spacegroup code shipped with Elk code version 4.3.6 (htp://elk.sourceforge.net), using space group Ia-3, all cell angles of 90.0 degrees and the following fractional coordinates of atoms (10.3390/ma7107059):

Lu1: $0.25,0.25,0.25$

Lu2: $0.46685,0.0,0.25$

O: $0.39159,0.15282,0.38011$

Positions of the rest of the atoms were generated by the symmetry operations respective to the space group. Note that Lu1 and Lu2 here are site labels, not to be confused with atom labels used below. The geometry from Spacegroup code was optimized using Quantum Espresso (see below) and shown in Table S2. Cell dimension was 19.432845 bohr.

The distances in the main text correspond to this defect-free optimized geometry (Table S2).

Table S2. The initial geometry used in the calculations (fractional coordinates).

\begin{tabular}{|c|c|c|c|c|c|c|c|}
\hline Atom label & fc1 & fc2 & fc3 & Atom label & fc1 & fc2 & fc3 \\
\hline Lu1 & 0.2499998 & 0.2499998 & 0.2499998 & O41 & 0.3912785 & 0.8476405 & 0.1200032 \\
\hline Lu2 & 0.7500002 & 0.7500002 & 0.7500002 & $\mathrm{O} 42$ & 0.8912793 & 0.3476402 & 0.6200032 \\
\hline Lu3 & 0.7500004 & 0.2500000 & 0.2499994 & $\mathrm{O} 43$ & 0.6087215 & 0.1523595 & 0.8799968 \\
\hline Lu4 & 0.2499996 & 0.7500000 & 0.7500006 & $\mathrm{O} 44$ & 0.1087207 & 0.6523598 & 0.3799968 \\
\hline Lu5 & 0.2499994 & 0.7500004 & 0.2500000 & $\mathrm{O} 45$ & 0.6087213 & 0.6523598 & 0.1200033 \\
\hline Lu6 & 0.7500006 & 0.2499996 & 0.7500000 & O46 & 0.1087210 & 0.1523596 & 0.6200034 \\
\hline Lu7 & 0.7500000 & 0.7500006 & 0.2499996 & O47 & 0.3912787 & 0.3476402 & 0.8799967 \\
\hline Lu8 & 0.2500000 & 0.2499994 & 0.7500004 & O48 & 0.8912790 & 0.8476404 & 0.3799966 \\
\hline Lu9 & 0.4664097 & 0.0000001 & 0.2499996 & O49 & 0.3799966 & 0.3912785 & 0.1523596 \\
\hline Lu10 & 0.9664106 & 0.4999998 & 0.7500001 & $\mathrm{O} 50$ & 0.8799967 & 0.8912790 & 0.6523597 \\
\hline Lu11 & 0.5335903 & -0.0000001 & 0.7500004 & O51 & 0.6200034 & 0.6087215 & 0.8476404 \\
\hline Lu12 & 0.0335894 & 0.5000002 & 0.2499999 & O52 & 0.1200033 & 0.1087210 & 0.3476403 \\
\hline Lu13 & 0.5335896 & 0.5000000 & 0.2500000 & O53 & 0.3799966 & 0.6087216 & 0.3476403 \\
\hline Lu14 & 0.0335890 & 0.0000003 & 0.7500004 & O54 & 0.8799966 & 0.1087207 & 0.8476408 \\
\hline Lu15 & 0.4664104 & 0.5000000 & 0.7500000 & O55 & 0.6200034 & 0.3912784 & 0.6523597 \\
\hline Lu16 & 0.9664110 & -0.0000003 & 0.2499996 & O56 & 0.1200034 & 0.8912793 & 0.1523592 \\
\hline Lu17 & 0.2499996 & 0.4664097 & 0.0000001 & O57 & 0.1200032 & 0.3912785 & 0.8476405 \\
\hline Lu18 & 0.7500001 & 0.9664106 & 0.4999998 & O58 & 0.6200032 & 0.8912793 & 0.3476402 \\
\hline Lu19 & 0.7500004 & 0.5335903 & -0.0000001 & O59 & 0.8799968 & 0.6087215 & 0.1523595 \\
\hline Lu20 & 0.2499999 & 0.0335894 & 0.5000002 & O60 & 0.3799968 & 0.1087207 & 0.6523598 \\
\hline Lu21 & 0.2500000 & 0.5335896 & 0.5000000 & O61 & 0.1200033 & 0.6087213 & 0.6523598 \\
\hline Lu22 & 0.7500004 & 0.0335890 & 0.0000003 & O62 & 0.6200034 & 0.1087210 & 0.1523596 \\
\hline Lu23 & 0.7500000 & 0.4664104 & 0.5000000 & O63 & 0.8799967 & 0.3912787 & 0.3476402 \\
\hline Lu24 & 0.2499996 & 0.9664110 & -0.0000003 & O64 & 0.3799966 & 0.8912790 & 0.8476404 \\
\hline Lu25 & 0.0000001 & 0.2499996 & 0.4664097 & O65 & 0.1523596 & 0.3799966 & 0.3912785 \\
\hline Lu26 & 0.4999998 & 0.7500001 & 0.9664106 & O66 & 0.6523597 & 0.8799967 & 0.8912790 \\
\hline Lu27 & -0.0000001 & 0.7500004 & 0.5335903 & O67 & 0.8476404 & 0.6200034 & 0.6087215 \\
\hline Lu28 & 0.5000002 & 0.2499999 & 0.0335894 & O68 & 0.3476403 & 0.1200033 & 0.1087210 \\
\hline Lu29 & 0.5000000 & 0.2500000 & 0.5335896 & O69 & 0.3476403 & 0.3799966 & 0.6087216 \\
\hline Lu30 & 0.0000003 & 0.7500004 & 0.0335890 & O70 & 0.8476408 & 0.8799966 & 0.1087207 \\
\hline Lu31 & 0.5000000 & 0.7500000 & 0.4664104 & O71 & 0.6523597 & 0.6200034 & 0.3912784 \\
\hline Lu32 & -0.0000003 & 0.2499996 & 0.9664110 & O72 & 0.1523592 & 0.1200034 & 0.8912793 \\
\hline O33 & 0.3912785 & 0.1523596 & 0.3799966 & O73 & 0.8476405 & 0.1200032 & 0.3912785 \\
\hline O34 & 0.8912790 & 0.6523597 & 0.8799967 & O74 & 0.3476402 & 0.6200032 & 0.8912793 \\
\hline 035 & 0.6087215 & 0.8476404 & 0.6200034 & O75 & 0.1523595 & 0.8799968 & 0.6087215 \\
\hline O36 & 0.1087210 & 0.3476403 & 0.1200033 & O76 & 0.6523598 & 0.3799968 & 0.1087207 \\
\hline O37 & 0.6087216 & 0.3476403 & 0.3799966 & O77 & 0.6523598 & 0.1200033 & 0.6087213 \\
\hline O38 & 0.1087207 & 0.8476408 & 0.8799966 & O78 & 0.1523596 & 0.6200034 & 0.1087210 \\
\hline O39 & 0.3912784 & 0.6523597 & 0.6200034 & O79 & 0.3476402 & 0.8799967 & 0.3912787 \\
\hline $\mathrm{O} 40$ & 0.8912793 & 0.1523592 & 0.1200034 & O80 & 0.8476404 & 0.3799966 & 0.8912790 \\
\hline
\end{tabular}




\section{FP-LAPW calculations}

Calculations with Elk 4.3.6 code used species files provided with the code. Only oxygen species file was modified - the local orbital with lorbl $=0$ and lorbord $=3$ was removed, resulting in total of two local orbitals with fixed linearization energies for oxygen local orbitals. The muffin-tin radius for Lu was 2.0 bohr, and 1.45 bohr for O. Other non-default options are specified in Table S3. Note that, due to the use of atomic units, electron charge in elk is +1 , while proton charge is -1 . Consequently, e.g. "chgexs -2" means two additional holes in the system, while "chgexs 1" means one additional electron. The values of rgkmax (defines maximum $|\mathbf{G}+\mathbf{k}|$ for APW functions, default is 7) and gmaxvr (maximum $|\mathbf{G}|$ for potential and density, default is 12) were increased in selected cases, in order to improve convergence. The k-point grid was $3 \times 3 \times 3$. Convergence threshold was twofold: root mean square change from last three iterations in Kohn-Sham potential was required to be lower than $1 \cdot 10^{-6}$ a.u., change in total energy was required to be lower than $1 \cdot 10^{-4}$ a.u.. Maximum angular momentum was 8 for APW functions, 7 for the outer part of muffin-tin and 3 for the inner part of muffin-tin.

Table S3. Selected settings used in Elk code.

\begin{tabular}{|c|c|c|c|}
\hline Variable name & Value & Variable name & Value \\
\hline tasks & 0,10 & isgkmax & -2 \\
\hline xctype & 1002099 & ngridk & 333 \\
\hline gmaxvr & See Table S5 & spinpol & $\mathrm{f} / \mathrm{t}$ \\
\hline rgkmax & See Table S5 & lradstp & 2 \\
\hline chgexs & $-2 /-1 / 0 / 1 / 2$ & dosmsum & $\mathrm{t}$ \\
\hline
\end{tabular}

The xctype option selects Räsänen, Pittalis and Proetto mGGA for exchange (209) and PZ correlation (9) from libxc (100).

\section{Convergence issues with Elk calculations}

While some of the two-vacancy (vacancy-vacancy pair) samples converged with no problems, some other repeatedly exhibited bad convergence. We have not found a reasonable explanation to it. Table S3 contains final parameters of the calculations, which allowed achieving convergence.

However, similar behavior was observed in some single-vacancy samples. $\mathrm{Lu}_{2} \mathrm{O}_{3}: \mathrm{V}_{\mathrm{O} 35}$ converged perfectly smoothly with total charges of $+2,+1,0,-1,-2$. On contrary, while closed-shell $\mathrm{Lu}_{2} \mathrm{O}_{3}: \mathrm{V}_{\text {Озз }}$ samples (total charges $+2,0,-2$ ) converged with minor issues, open-shell $\mathrm{Lu}_{2} \mathrm{O}_{3}: \mathrm{V}_{\text {Озз }}$ samples (total charges $-1,+1$ ) did not converge, despite many attempts and different tricks. Such a thing is not supposed to happen, given the fact that all of the oxygen atoms in the structure must be identical. We have performed a test where the cell was centered at either O33 or O35 (i.e. all atoms of the cell were translated such that the center atom had fractional coordinates of $0,0,0)$, and the fractional coordinates of the rest of the atoms were wrapped to the $[-0.5,0.5)$ range (i.e. the new fractional coordinate $f c^{\prime}$ was equal to $f c-1$ if $f c$ was $\geq 0.5$, and to $f c+1$ if $f c$ was $<-0.5$, $f c^{\prime}$ being the fractional coordinate after re-centering). The two resulting geometries were identical, as they should be, given the fact that all oxygen atoms in cubic $\mathrm{Lu}_{2} \mathrm{O}_{3}$ belong to the same crystallographic position. Thus, there was no error in the initial geometry.

The bad convergence issue often manifested as a problem in finding linearization energies for some of the Lu atoms. The problem in known in the Elk community and is discussed at the respective online forum (https://sourceforge.net/p/elk/discussion/897820/). There is no definite simple answer to why does it happen, as well as there is no universal solution. Typical causes are: bad geometry, low basis, wrong muffin-tin radii. Typical solutions include: changes in geometry, increase in basis (rgkmax and gmaxvr). Sometimes, it is impossible to change muffin-tin radius due to short bonds. Small radii may result in rather deep-lying (large negative) linearization energies. In that case, increased range of search for the converging value of linearization energy is used (via setting demaxbnd to 5 and more). In our case, such changes in parameters were not helpful.

However, later, we were able to converge the problematic calculations using Elk 6.3.2, Broyden mixing (mixtype $=3$ ) and broydpm of 0.1, 0.0375. Noteworthy, using the same parameters in Elk 4.3.6 did not solve the problem. 


\section{Linearization energy cloning}

The species files contain initial guess values of linearization energies, obtained from single-atom calculations. The values typically change quite significantly during the calculations. The idea of the trick was, for a particular local orbital, take the average of that orbital linearization energies from all of the Lu atoms from a converged calculation, and apply it as the initial guess value in the problematic calculation. Such approach, combined with increased basis, have solved some of the convergence issues.

\section{Vacancy site selection}

In order to create structures with oxygen vacancies, selected oxygen atoms were removed. For the single-vacancy structures, either O33 or O35 was removed (See Table S4). For the structures with two vacancies, O33 was removed, while the second atom to be removed was selected accordingly to the distance between it and O33. In the table below, $\mathrm{R}_{33}$ is such a distance.

$\mathrm{R}_{33}$ of atom $\mathrm{X}$ can be measured directly between atom $\mathrm{X}$ and O33, within a single cell, or between symmetry reflections of atom $X$ in the adjacent cells. True $R_{33}$ is the shortest one of these. The xPB parameter in the table indicates if the respective distance was measured across cells (i.e. trough periodic boundary, $\mathrm{PB}$ ). In other words, the shortest line segment connecting atom $\mathrm{X}$ and O33 may lay within a single unit cell (xPB is "no"), or not (xPB is “yes”).

In several cases, two oxygen atoms were characterized by the same $\mathrm{R}_{33}$, while only for one of them $\mathrm{R}_{33}$ was measured within a single sell; such an atom was selected. The other one was discarded due to periodic boundary condition, PBC. $\Delta$ is the difference between a particular $R_{33}$ and the previous $\mathrm{R}_{33}$.

After the selection process, and removal of the respective atoms, the geometries of the structures were relaxed using Quantum Espresso.

\section{Pseudopotential testing}

Before use, the pseudopotentials (PPs) has been substantially tested and compared to PSLibrary (10.1016/j.commatsci.2014.07.043) Lu (4f in core) and O USPPs. An unoptimized unit cell of $\mathrm{Lu}_{2} \mathrm{O}_{3}$ (as prepared by Spacegroup) was the test subject. In order to facilitate the tests, two kinds of comparison were performed.

In the first test, the displacements of atoms (in respect to the initial positions) due to geometry optimization were analyzed. Assuming the experimental geometry as correct, the pseudopotential resulting in smaller changes is considered better. Both sets were tested with plane wave and kinetic energy cutoffs or either 40, 400 or 60,600 a.u.. Two kinds of relaxation were performed, atomic positions only or full (atomic positions and cell vectors). It turned out, that the results from different cutoff parameters were very similar. For the 40, 400 cutoffs and positions-only relaxation, root mean square displacement in fractional coordinates for GBRV potentials was 0.00105 , while for PSLibrary potentials it was 0.00297 . For the 40,400 cutoffs and full relaxation, root mean square displacement in fractional coordinates for GBRV potentials was 0.00050 , while for PSLibrary potentials it was 0.00063 . According to this test, the GBRV potentials were deemed better.

In the second test, cell parameter response to optimization was analyzed. With Quantum Espresso, a relaxation of cell vectors only was performed, again with the two sets of cutoff parameters. Data from the full relaxation above was also used. The resulting changes in cell dimensions (as percentage of change in respect to the initial / experimental values) were compared to the respective data from Elk (with parameters as described above, except for LDA as density functional). With Elk, equation of state (EOS) calculations were performed on the cell: a series of total energy calculations with different scaling factors $(0.95-1.05$, with the step of 0.01$)$. The respective $\mathrm{E}=\mathrm{f}(\mathrm{scale})$ curve was fitted with Murnaghan equation of state (10.1073/pnas.30.9.244). From the fit, optimal value of the cell dimension was obtained and used as a reference. According to the EOS, the Perdew-Zunger optimized cell dimension of $\mathrm{Lu}_{2} \mathrm{O}_{3}$ cell is 19.301670 bohr. The PSLibrary PPs resulted in the cells 1.63-1.66\% smaller than the Elk value. The GBRV resulted in the cells $0.66-0.69 \%$ larger than the Elk value (and closer to experimental value), and were deemed better due to the smaller difference. 
Table S4. Distances between O33 and the other oxygen atoms, as well as selection conditions.

\begin{tabular}{|c|c|c|c|c|c|c|}
\hline Index of oxygen atom & $\mathrm{R}_{33}, \AA$ & $\mathrm{xPB}$ & $\Delta, \AA$ & $\Delta>0.05 \AA$ & Selection result & Reason not to select \\
\hline 33 & 0 & No & & & & \\
\hline 60 & 2.874 & No & 2.874 & Yes & Yes & \\
\hline 79 & 2.874 & Yes & 0.000 & No & No & Same $\mathrm{R}_{33}(\Delta=0)$ \\
\hline 52 & 2.882 & No & 0.008 & No & No & $\Delta$ too small \\
\hline 68 & 2.882 & No & 0.000 & No & No & Same $\mathrm{R}_{33}$ \\
\hline 37 & 3.026 & No & 0.144 & Yes & Yes & \\
\hline 62 & 3.38 & No & 0.354 & Yes & Yes & \\
\hline 69 & 3.38 & No & 0.000 & No & No & Same $\mathrm{R}_{33}$ \\
\hline 49 & 3.428 & No & 0.048 & Assumed yes & Yes & \\
\hline 65 & 3.428 & No & 0.000 & No & No & Same $\mathrm{R}_{33}$ \\
\hline 77 & 3.622 & No & 0.194 & Yes & Yes & \\
\hline 58 & 3.622 & Yes & 0.000 & No & No & PBC \\
\hline 46 & 3.857 & No & 0.235 & Yes & Yes & \\
\hline 41 & 4.172 & Yes & 0.315 & Yes & Yes & \\
\hline 55 & 4.452 & No & 0.280 & Yes & Yes & \\
\hline 75 & 4.452 & Yes & 0.000 & No & No & Same $\mathrm{R}_{33}$ \\
\hline 36 & 4.479 & No & 0.027 & No & No & $\Delta$ too small \\
\hline 76 & 4.574 & No & 0.095 & Yes & Yes & \\
\hline 56 & 4.574 & Yes & 0.000 & No & No & PBC \\
\hline 35 & 4.624 & Yes & 0.050 & No & Yes & $\Delta$ too small \\
\hline 53 & 4.749 & No & 0.125 & Yes & Yes & \\
\hline 73 & 4.749 & No & 0.000 & No & No & Same $\mathrm{R}_{33}$ \\
\hline 71 & 5.563 & No & 0.814 & Yes & Yes & \\
\hline 64 & 5.563 & Yes & 0.000 & No & No & PBC \\
\hline 47 & 5.575 & Yes & 0.012 & No & No & $\Delta$ too small \\
\hline 63 & 5.662 & No & 0.087 & Yes & Yes & \\
\hline 72 & 5.662 & Yes & 0.000 & No & No & Same $\mathrm{R}_{33}$ \\
\hline 43 & 5.664 & Yes & 0.002 & No & No & $\Delta$ too small \\
\hline 39 & 5.763 & Yes & 0.099 & Yes & Yes & \\
\hline 40 & 5.858 & Yes & 0.095 & Yes & Yes & \\
\hline 44 & 5.972 & Yes & 0.114 & Yes & Yes & \\
\hline 48 & 6.091 & Yes & 0.119 & Yes & Yes & \\
\hline 42 & 6.107 & Yes & 0.016 & No & No & $\Delta$ too small \\
\hline 57 & 6.140 & No & 0.033 & No & Yes & \\
\hline 78 & 6.140 & No & 0.000 & No & No & Same $\mathrm{R}_{33}$ \\
\hline 61 & 6.199 & No & 0.059 & Yes & Yes & \\
\hline 70 & 6.199 & Yes & 0.000 & No & No & Same $\mathrm{R}_{33}$ \\
\hline 45 & 6.277 & Yes & 0.078 & Yes & Yes & \\
\hline 50 & 6.417 & Yes & 0.140 & Yes & Yes & \\
\hline 66 & 6.417 & Yes & 0.000 & No & No & Same $\mathrm{R}_{33}$ \\
\hline 38 & 6.764 & Yes & 0.347 & Yes & Yes & \\
\hline 54 & 7.040 & No & 0.276 & Yes & Yes & \\
\hline 74 & 7.040 & Yes & 0.000 & No & No & Same $\mathrm{R}_{33}$ \\
\hline 51 & 7.184 & No & 0.144 & Yes & Yes & \\
\hline 67 & 7.184 & No & 0.000 & No & No & Same $\mathrm{R}_{33}$ \\
\hline 59 & 7.333 & No & 0.149 & Yes & Yes & \\
\hline 80 & 7.333 & Yes & 0.000 & No & No & Same $\mathrm{R}_{33}$ \\
\hline 34 & 9.001 & Yes & 1.668 & Yes & Yes & \\
\hline
\end{tabular}

Distances in this table were measured in the experimental geometry 


\section{Elk parameters for the vacancy-vacancy structures}

The calculations were performed in two batches. Most work was done using Elk 4.3.6. Some samples exhibited problematic convergence, while some other did not converge, despite various efforts $\left(\mathrm{Lu}_{2} \mathrm{O}_{3}:\left(V_{\mathrm{O} 33}, V_{\mathrm{O} 34}\right)^{\bullet \bullet}, \mathrm{Lu}_{2} \mathrm{O}_{3}:\left(V_{\mathrm{O} 33}, V_{\mathrm{O} 39}\right)^{\boldsymbol{\bullet}}, \mathrm{Lu}_{2} \mathrm{O}_{3}:\left(V_{\mathrm{O} 33}, V_{\mathrm{O} 40}\right)^{\bullet \bullet}\right.$,

$\left.\mathrm{Lu}_{2} \mathrm{O}_{3}:\left(V_{\mathrm{O} 33}, V_{\mathrm{O} 48}\right)^{\bullet \bullet}, \mathrm{Lu}_{2} \mathrm{O}_{3}:\left(V_{\mathrm{O} 33}, V_{\mathrm{O} 59}\right)^{\bullet \bullet}\right)$. Later, some of the calculations were repeated using Elk 6.3.2. In this second batch, we have managed to converge the problematic calculations using Broyden mixing (mixtype $=3$ ) and broydpm of 0.1, 0.0375 .

Unless specified otherwise, the calculations used Elk 4.3.6 with rgkmax $=7.0$ and gmaxvr $=12$. For problematic samples, basis was increased and trimming of small components of exchangecorelation potential Fourier transform was enabled (trimvg option). In some cases, linearization energy cloning was applied. The details are shown in Table S5.

In the case of $\mathrm{Lu}_{2} \mathrm{O}_{3}: V_{\mathrm{O} 33}{ }^{\bullet}, V_{\mathrm{O}}^{\bullet \bullet}$ samples, linearization energy cloning from the respective $\mathrm{Lu}_{2} \mathrm{O}_{3}: V_{\mathrm{O} 33}^{\times}, V_{\mathrm{O}}^{\times}$. was initially applied to all of the samples, and then reverted for selected ones that did not converge, see Table S5.

Table S5. Elk parameters for the samples that experienced convergence troubles.

\begin{tabular}{|c|c|c|c|c|}
\hline Sample & rgkmax & gmaxvr & trimvg & If l.e. were cloned \\
\hline \multicolumn{5}{|c|}{ Elk 4.3.6 } \\
\hline $\mathrm{Lu}_{2} \mathrm{O}_{3}: V_{\mathrm{O} 33}^{\times}$ & 7.0 & 12 & false & yes \\
\hline $\mathrm{Lu}_{2} \mathrm{O}_{3}: V_{\mathrm{O} 33}^{\bullet}$ & 8.0 & 18 & true & no \\
\hline $\mathrm{Lu}_{2} \mathrm{O}_{3}: V_{\mathrm{O} 33}^{\prime \prime}$ & 8.0 & 18 & true & no \\
\hline $\mathrm{Lu}_{2} \mathrm{O}_{3}: V_{\mathrm{O} 33}^{\times}, V_{\mathrm{O} 46}^{\times}$ & 7.5 & 18 & false & yes \\
\hline $\mathrm{Lu}_{2} \mathrm{O}_{3}:\left(V_{\mathrm{O} 33}, V_{\mathrm{O} 38}\right)^{\bullet \bullet}$ & 7.5 & 15 & true & yes \\
\hline $\mathrm{Lu}_{2} \mathrm{O}_{3}:\left(V_{\mathrm{O} 33}, V_{\mathrm{O} 44}\right)^{\bullet \bullet}$ & 7.5 & 15 & true & no \\
\hline $\mathrm{Lu}_{2} \mathrm{O}_{3}:\left(V_{\mathrm{O} 33}, V_{\mathrm{O} 45}\right)^{\bullet \bullet}$ & 7.5 & 15 & false & no \\
\hline $\mathrm{Lu}_{2} \mathrm{O}_{3}:\left(V_{\mathrm{O} 33}, V_{\mathrm{O} 46}\right)^{\bullet \bullet}$ & 8.5 & 18 & true & no \\
\hline $\mathrm{Lu}_{2} \mathrm{O}_{3}:\left(V_{\mathrm{O} 33}, V_{\mathrm{O} 50}\right)^{\bullet \bullet}$ & 7.5 & 15 & false & no \\
\hline $\mathrm{Lu}_{2} \mathrm{O}_{3}:\left(V_{\mathrm{O} 33}, V_{\mathrm{O} 51}\right)^{\bullet \bullet}$ & 8.0 & 18 & true & yes \\
\hline $\mathrm{Lu}_{2} \mathrm{O}_{3}:\left(V_{\mathrm{O} 33}, V_{\mathrm{O} 54}\right)^{\bullet \bullet}$ & 7.5 & 15 & true & yes \\
\hline $\mathrm{Lu}_{2} \mathrm{O}_{3}:\left(V_{\mathrm{O} 33}, V_{\mathrm{O} 61}\right)^{\bullet \bullet}$ & 8.0 & 18 & true & no \\
\hline $\mathrm{Lu}_{2} \mathrm{O}_{3}:\left(V_{\mathrm{O} 33}, V_{\mathrm{O} 63}\right)^{\bullet \bullet}$ & 7.5 & 15 & false & no \\
\hline $\mathrm{Lu}_{2} \mathrm{O}_{3}:\left(V_{\mathrm{O} 33}, V_{\mathrm{O} 71}\right)^{\bullet \bullet}$ & 7.5 & 18 & true & yes \\
\hline $\mathrm{Lu}_{2} \mathrm{O}_{3}: V_{\mathrm{O} 33}^{\bullet \bullet}, V_{\mathrm{O} 41}^{\bullet \bullet}$ & 8.0 & 18 & true & no \\
\hline $\mathrm{Lu}_{2} \mathrm{O}_{3}: V_{\mathrm{O} 33}^{\bullet \bullet}, V_{\mathrm{O} 54}^{\bullet \bullet}$ & 8.0 & 18 & true & no \\
\hline $\mathrm{Lu}_{2} \mathrm{O}_{3}: V_{\mathrm{O} 33}^{\bullet \bullet}, V_{\mathrm{O} 55}^{\bullet \bullet}$ & 8.0 & 18 & true & no \\
\hline $\mathrm{Lu}_{2} \mathrm{O}_{3}: V_{\mathrm{O} 33}^{\bullet \bullet}, V_{\mathrm{O} 62}^{\bullet \bullet}$ & 7.0 & 12 & false & no \\
\hline \multicolumn{5}{|c|}{ Elk 6.3.2 } \\
\hline $\mathrm{Lu}_{2} \mathrm{O}_{3}:\left(V_{\mathrm{O} 33}, V_{\mathrm{O} 34}\right)^{\bullet \bullet}$ & 7.0 & 12 & false & no \\
\hline $\mathrm{Lu}_{2} \mathrm{O}_{3}:\left(V_{\mathrm{O} 33}, V_{\mathrm{O} 39}\right)^{\bullet \bullet}$ & 7.0 & 12 & false & no \\
\hline $\mathrm{Lu}_{2} \mathrm{O}_{3}:\left(V_{\mathrm{O} 33}, V_{\mathrm{O} 40}\right)^{\bullet \bullet}$ & 7.8 & 17 & false & no \\
\hline $\mathrm{Lu}_{2} \mathrm{O}_{3}:\left(V_{\mathrm{O} 33}, V_{\mathrm{O} 48}\right)^{\bullet \bullet}$ & 7.0 & 12 & false & no \\
\hline $\mathrm{Lu}_{2} \mathrm{O}_{3}:\left(V_{\mathrm{O} 33}, V_{\mathrm{O} 59}\right)^{\bullet \bullet}$ & 7.0 & 12 & false & no \\
\hline
\end{tabular}




\section{Frenkel pair formation}

In order to form a Frenkel pair, oxygen vacancy at O33 position was created (O33 was removed), and an interstitial oxygen at one of the anionic void position was added (Table S6). In order to generate the anionic void positions, another atom was added to the Spacegroup input, at fractional coordinates of $0.375,0.375,0.375$. Total of 16 positions were generated, and the corresponding 16 structures with a Frenkel defect were analyzed. The geometries of the structures were optimized. Next, Elk energy and DOS calculations were performed, as described above.

\section{DOS peak fitting}

The DOS plot peaks were fitted with a sum of Gaussian functions, as shown in Fig. S1. The initial guess was composed of peaks with $0.001 \mathrm{Ha}$ width and 1000 states per Ha per unit cell height, roughly at the target peak position. The fitting was performed using custom software in Python, which used SciPy module. The fitting used scipy.optimize.least_squares least squares problem solver, with default Trust Region Reflective minimization algorithm. Peak positions, widths and heights were variational parameters. The fitting region included a small fragment ( $\sim 0.005$ Ha wide) of valence band edge, a somehow larger fragment of conduction band edge, and the range between the bands.

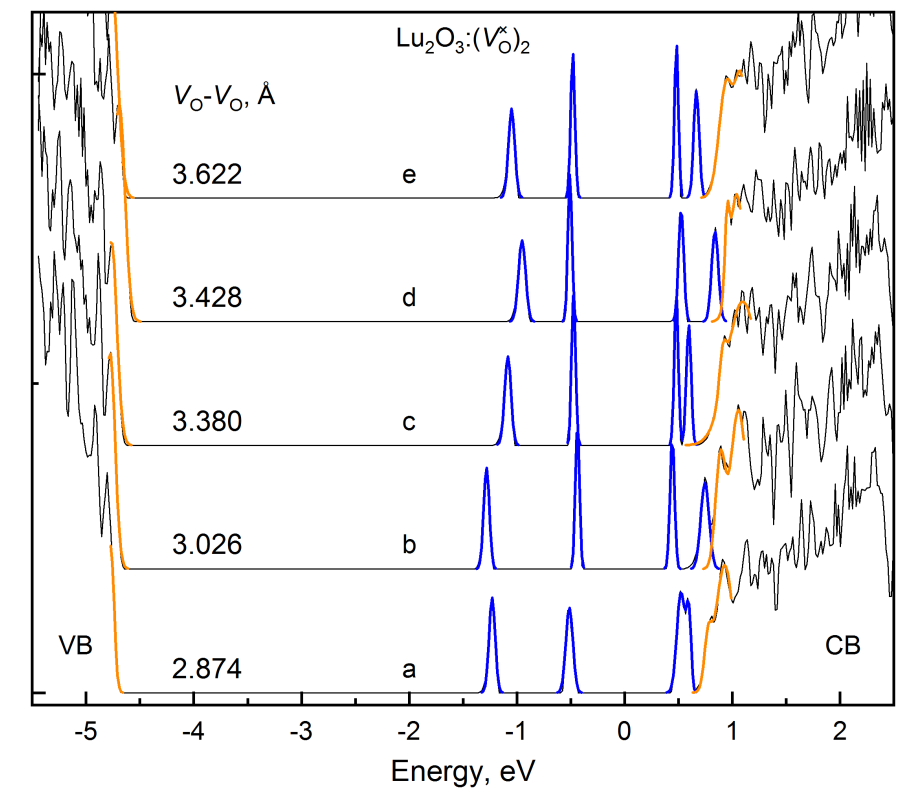

Fig. S1. An example of DOS peak fitting with Gaussian functions.

$\mathrm{VB}$ is valence band, CB is conduction band.

Defect peaks are in blue, band edges are orange.

In Fig. S1, for visualization purposes, each Gaussian is plotted separately (the actual fitting fitting used their sum). It is clear that 4 peaks correspond to each oxygen vacancy-vacancy pair. Sometimes, one of the peaks partially overlaps with conduction band (Fig. S1 b,d). Sometimes, two of the peaks overlap with each other (Fig. S1 a). Still, it is possible to distinguish the defect peaks from the band. Due to possible overlaps, total of 7 peaks were used in the fitting: one for valence band, four for the defects and two for conduction band. The fitting results are available in the Lu2O3_2Vo_Supplementary_Tables.xls spreadsheet. 

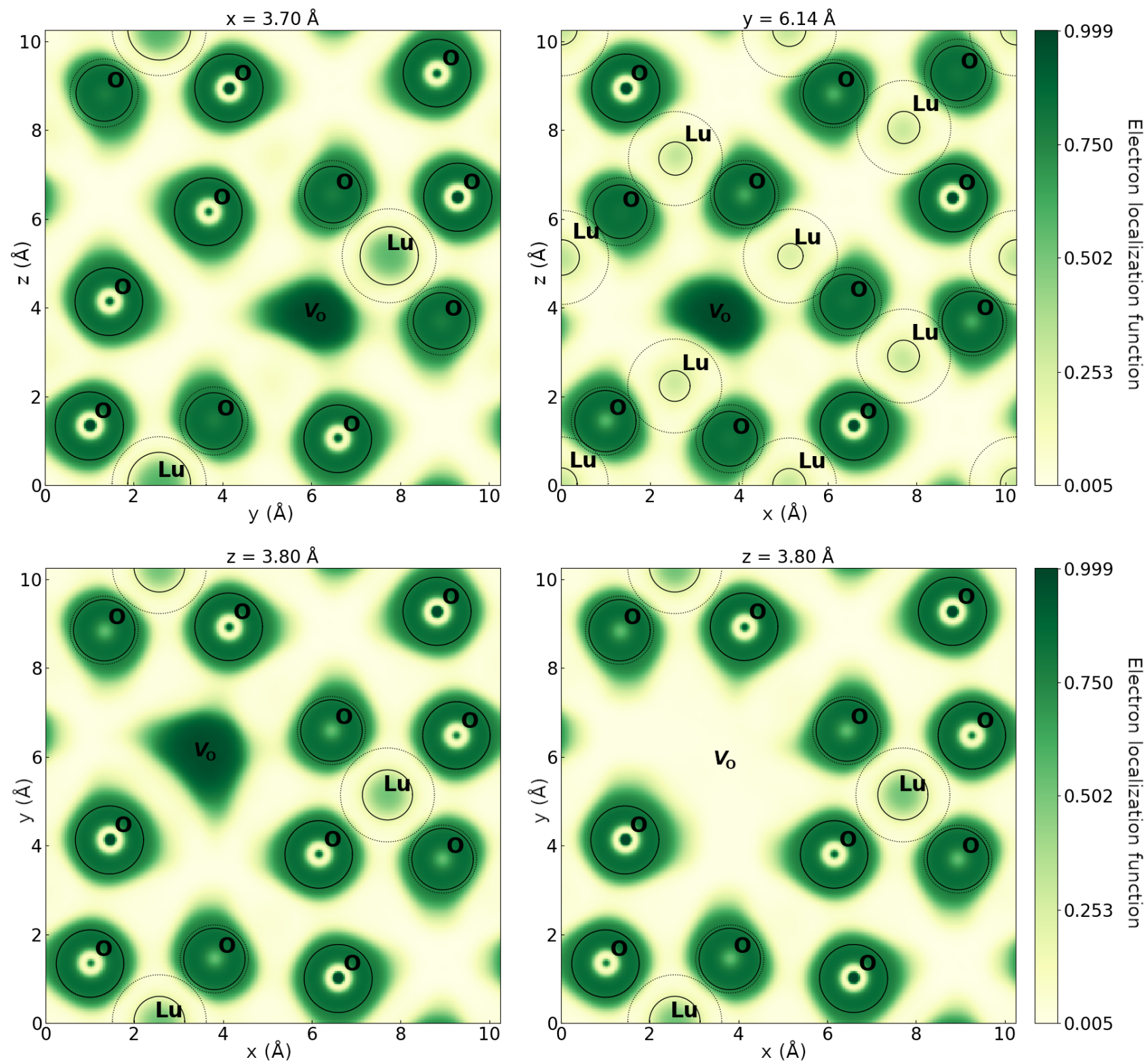

Fig. S2. Electron localization function plots (in the planes that contained the removed oxygen atom) for $\mathrm{Lu}_{2} \mathrm{O}_{3}: V_{\mathrm{O} 35}^{\times}$(in the $y z, x z$ and $x y$ planes) and $\mathrm{Lu}_{2} \mathrm{O}_{3}: V_{\mathrm{O} 35}^{\times}$without the trapped electrons (in $x y$ plane)

\section{Note on atomic basis shift}

Elk code applies automatic atomic basis shift. All of the atoms in the cell are translated by a certain vector and then each fractional coordinate is wrapped to the $[0,1)$ range. From Elk manual:

[subroutine findsymcrys] In order to determine the translation vectors, the entire atomic basis is shifted so that the first atom in the smallest set of atoms of the same species is at the origin. Then all displacement vectors between atoms in this set are checked as possible symmetry translations.

The resulting geometry is physically the same system, but the particular atoms have different positions. In the calculations, the shift was about $-0.25,-0.25,-0.25$ in fractional coordinates. All of the ELF plots correspond to the shifted geometry, not the input geometry. I.e. the ELF atomic positions are approximately those from Table S2, plus the shift. 


\section{Vacancy-vacancy pairs - DOS plots}

The DOS plots of $\mathrm{Lu}_{2} \mathrm{O}_{3}: V_{\mathrm{O} 33}^{\times}, V_{\mathrm{O} . .}^{\times}\left(\mathrm{Lu}_{2} \mathrm{O}_{3}: 2 V_{\mathrm{O}}^{\times}\right), \mathrm{Lu}_{2} \mathrm{O}_{3}:\left(V_{\mathrm{O} 33}, V_{\mathrm{O} . . .}\right)^{\bullet \bullet}$ and $\mathrm{Lu}_{2} \mathrm{O}_{3}: V_{\mathrm{O} 33}^{\bullet \bullet}, V_{\mathrm{O} .}^{\bullet \bullet}$ $\left(\mathrm{Lu}_{2} \mathrm{O}_{3}: 2 V_{\mathrm{O}}^{\bullet \bullet}\right)$ were quite similar, and differed mostly in Fermi level position. Thus, only $\mathrm{Lu}_{2} \mathrm{O}_{3}:\left(V_{\mathrm{O} 33}, V_{\mathrm{O} . .}\right)^{\bullet \bullet}$ plots were shown in the main text. The rest are shown below.

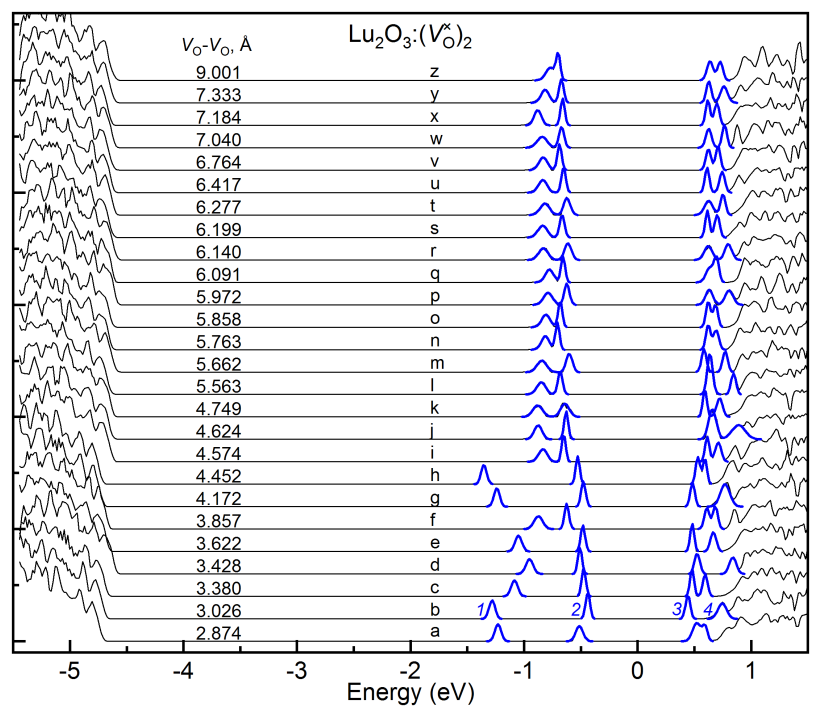

Fig. S3. DOS plots of $\mathrm{Lu}_{2} \mathrm{O}_{3}: V_{\mathrm{O} 33}^{\times}, V_{\mathrm{O}}^{\times}$. structures. Fermi level is at zero.

The Gaussian fits are shown in blue.

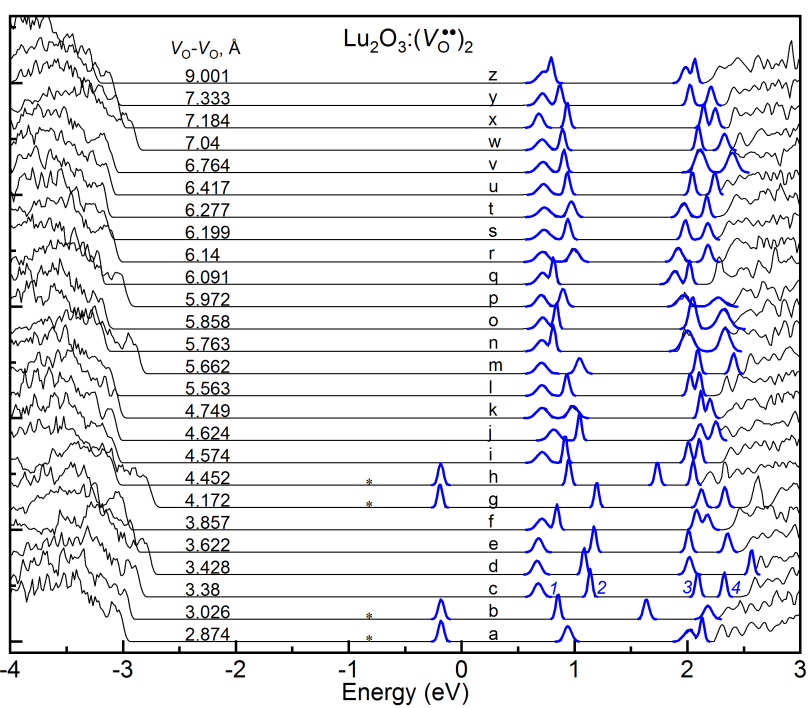

Fig. S4. DOS plots of $\mathrm{Lu}_{2} \mathrm{O}_{3}: V_{\mathrm{O} 33}^{\bullet \bullet}, V_{\mathrm{O}}^{\bullet \bullet}$ structures. Fermi level is at zero, except for a,b,g,h, which were shifted left to match their valence band positions with the rest of the curves.

Asterisks show Fermi level positions of the shifted curves. The Gaussian fits are shown in blue.

In comparison to $\mathrm{Lu}_{2} \mathrm{O}_{3}: V_{\mathrm{O}}$, for both deep and shallow traps in $\mathrm{Lu}_{2} \mathrm{O}_{3}: 2 V_{\mathrm{O}}^{\times}$, there are two defectrelated peaks instead of one. In other words, total of four peaks correspond to the two oxygen vacancies (Figs. S3, S4). The shallow trap peaks are at proximity of CB and overlap with it in some cases (e.g. Fig. S3 b, d, g, p, r, w). The defect peak splitting is the smallest at the largest distance between the vacancies (Fig. S3 z).

In most of the samples in Fig. S4, Fermi level was located about $3 \mathrm{eV}$ above VB. However, several samples were characterized by very high splitting of the defect peaks, resulting in shift of Fermi level down to about $2 \mathrm{eV}$ above VB. In Fig. S4, the respective curves (a, b, g, h) were shifted left by 0.03 Hartree $(\sim 0.82 \mathrm{eV})$ so that their VB and CB positions matched the rest of the series. The position of Fermi level of these samples is marked by asterisks. Somewhat larger gap between the two "deep" peaks of the vacancies is observed in this case. For $\mathrm{Lu}_{2} \mathrm{O}_{3}: 2 V_{\mathrm{O}}^{\bullet \bullet}$, the defect peak splitting is also the smallest at the largest distance between the vacancies (Fig. S3 z).

\section{Vacancy-vacancy pairs - spatial properties}

One of the hypotheses explaining the larger defect-defect energy difference in DOS was that mutual spatial orientations of the vacancies might be the cause. In order to analyze the spatial properties of the vacancies, the following steps were applied. Take a OLu ${ }_{4}$ cluster (local symmetry $\mathrm{C}_{1}$ ) in the unperturbed geometry. First, rotate the $\mathrm{OLu}_{4}$ cluster in such a way that its longest bond is aligned along $z$ axis. Then, rotate it around $z$ axis so that the shortest bond lies within $x z$ plane. There would be "left" and "right" vacancies, that differ by reflection at $x z$ plane. The vacancy sites are thus chiral. However, we have found no correlations between the chirality (right-right, right-left and left-left pairs) and the properties of DOS. 
Different lengths of bonds in the $\mathrm{OLu}_{4}$ cluster mean that cluster barycenter (calculated with $\mathrm{Lu}$ atoms only) is not at the oxygen position. The oxygen-barycenter vector can thus be used to characterize spatial orientation of a cluster. Turns out that mutual spatial orientations (in a form of angle between the two orientation vectors) do not correlate with the DOS features (larger splitting). The length of the oxygen-barycenter vector is the same for all of the vacancies.

\section{Vacancy-vacancy pairs - trap depths and transition energies}

Electron trap depth for empty trap is an impractical value - filled trad depth might be different and is the one that counts. Thus, for empty defect bands, wavelengths of transitions they might be involved in are shown. The wavelengths correspond to the respective energy differences calculated from DOS using peak-to-peak approach mentioned above. These values are visualized in Fig. 14 of the main text.

The vacancy-vacancy distance $\mathrm{R}_{V-V}$ in the table is, in fact, the oxygen-oxygen distance for the removed atoms, with correspondence to Table S4.

Table S6. Trap depths and wavelengths of possible transitions from conduction band

\begin{tabular}{|c|c|c|c|c|c|c|c|c|c|}
\hline \multirow{2}{*}{$\mathrm{R}_{V-V}, \AA$} & \multicolumn{6}{|c|}{ Trap depths, by the number of band, eV } & \multicolumn{3}{|c|}{ Wavelengths, nm } \\
\hline & $1 \rightarrow 3$ & $1 \rightarrow 4$ & $1 \rightarrow \mathrm{CB}$ & $2 \rightarrow 3$ & $2 \rightarrow 4$ & $2 \rightarrow \mathrm{CB}$ & $\mathrm{VB} \rightarrow 3$ & $\mathrm{VB} \rightarrow 4$ & $\mathrm{VB} \rightarrow \mathrm{CB}$ \\
\hline 2.874 & 1.75 & 1.83 & 2.00 & 1.04 & 1.11 & 1.29 & 234 & 231 & 224 \\
\hline 3.026 & 1.72 & 2.03 & 2.17 & 0.88 & 1.18 & 1.33 & 237 & 224 & 219 \\
\hline 3.38 & 1.56 & 1.68 & 2.00 & 0.95 & 1.07 & 1.39 & 236 & 231 & 218 \\
\hline 3.428 & 1.48 & 1.79 & 1.90 & 1.03 & 1.35 & 1.46 & 238 & 224 & 220 \\
\hline 3.622 & 1.53 & 1.72 & 1.99 & 0.96 & 1.15 & 1.43 & 237 & 228 & 217 \\
\hline 3.857 & 1.48 & 1.56 & 1.72 & 1.23 & 1.31 & 1.48 & 232 & 229 & 222 \\
\hline 4.172 & 1.72 & 2.01 & 2.21 & 0.96 & 1.25 & 1.45 & 237 & 225 & 217 \\
\hline 4.452 & 1.88 & 1.95 & 2.18 & 1.06 & 1.12 & 1.35 & 232 & 230 & 220 \\
\hline 4.574 & 1.45 & 1.54 & 1.69 & 1.26 & 1.36 & 1.50 & 233 & 229 & 223 \\
\hline 4.624 & 1.54 & 1.77 & 1.85 & 1.29 & 1.52 & 1.60 & 231 & 222 & 219 \\
\hline 4.749 & 1.47 & 1.60 & 1.89 & 1.23 & 1.36 & 1.65 & 234 & 229 & 217 \\
\hline 5.563 & 1.49 & 1.69 & 1.86 & 1.32 & 1.53 & 1.70 & 232 & 223 & 216 \\
\hline 5.662 & 1.43 & 1.61 & 1.76 & 1.19 & 1.37 & 1.52 & 235 & 227 & 221 \\
\hline 5.763 & 1.43 & 1.50 & 1.72 & 1.33 & 1.40 & 1.61 & 233 & 230 & 221 \\
\hline 5.858 & 1.43 & 1.49 & 1.74 & 1.30 & 1.37 & 1.61 & 232 & 230 & 220 \\
\hline 5.972 & 1.42 & 1.60 & 1.75 & 1.25 & 1.43 & 1.58 & 233 & 226 & 220 \\
\hline 6.091 & 1.41 & 1.47 & 1.72 & 1.30 & 1.35 & 1.60 & 232 & 229 & 219 \\
\hline 6.14 & 1.45 & 1.63 & 1.76 & 1.24 & 1.41 & 1.54 & 233 & 226 & 221 \\
\hline 6.199 & 1.45 & 1.54 & 1.71 & 1.28 & 1.37 & 1.54 & 233 & 229 & 222 \\
\hline 6.277 & 1.44 & 1.57 & 1.83 & 1.25 & 1.37 & 1.64 & 233 & 228 & 217 \\
\hline 6.417 & 1.45 & 1.58 & 1.81 & 1.26 & 1.40 & 1.63 & 233 & 227 & 218 \\
\hline 6.764 & 1.46 & 1.54 & 1.76 & 1.31 & 1.40 & 1.62 & 232 & 228 & 220 \\
\hline 7.04 & 1.47 & 1.61 & 1.72 & 1.30 & 1.44 & 1.55 & 233 & 227 & 222 \\
\hline 7.184 & 1.50 & 1.58 & 1.79 & 1.28 & 1.36 & 1.57 & 232 & 229 & 220 \\
\hline 7.333 & 1.44 & 1.58 & 1.71 & 1.30 & 1.43 & 1.57 & 232 & 227 & 221 \\
\hline 9.001 & 1.34 & 1.43 & 1.65 & 1.41 & 1.49 & 1.72 & 232 & 228 & 219 \\
\hline Min. deviation: & -0.17 & -0.22 & -0.19 & -0.32 & -0.27 & -0.25 & -2 & -6 & -3 \\
\hline Average values: & 1.51 & 1.65 & 1.84 & 1.20 & 1.34 & 1.53 & 234 & 227 & 220 \\
\hline Max. deviation: & 0.38 & 0.38 & 0.37 & 0.21 & 0.19 & 0.19 & 4 & 4 & 4 \\
\hline
\end{tabular}


Table S7. Trap depths and wavelengths of possible transitions in $\mathrm{Lu}_{2} \mathrm{O}_{3}:\left(V_{\mathrm{O} 33}, V_{\mathrm{O} . .}\right)$

\begin{tabular}{|c|c|c|c|c|c|c|c|c|}
\hline \multirow{2}{*}{$\mathrm{R}_{V-V}, \AA$} & \multicolumn{4}{|c|}{ Trap depths, by the bands, eV } & \multicolumn{4}{|c|}{ Wavelengths, by the bands, nm } \\
\hline & $1 \rightarrow 2$ & $1 \rightarrow 3$ & $1 \rightarrow 4$ & $1 \rightarrow \mathrm{CB}$ & $\mathrm{VB} \rightarrow 2$ & $\mathrm{VB} \rightarrow 3$ & $\mathrm{VB} \rightarrow 4$ & $\mathrm{VB} \rightarrow \mathrm{CB}$ \\
\hline 2.874 & 0.91 & 2.00 & 2.08 & 2.31 & 301 & 238 & 234 & 225 \\
\hline 3.026 & 1.04 & 1.92 & 2.34 & 2.50 & 296 & 245 & 226 & 220 \\
\hline 3.38 & 0.62 & 1.61 & 1.82 & 2.18 & 308 & 247 & 237 & 222 \\
\hline 3.428 & 0.46 & 1.54 & 1.98 & 2.10 & 314 & 247 & 227 & 222 \\
\hline 3.622 & 0.57 & 1.54 & 1.85 & 2.13 & 307 & 247 & 233 & 221 \\
\hline 3.857 & 0.20 & 1.55 & 1.65 & 1.87 & 318 & 236 & 232 & 223 \\
\hline 4.172 & 0.88 & 1.92 & 2.22 & 2.29 & 299 & 239 & 226 & 224 \\
\hline 4.452 & 0.95 & 2.11 & 2.19 & 2.45 & 304 & 237 & 233 & 223 \\
\hline 4.574 & 0.17 & 1.49 & 1.57 & 1.81 & 316 & 237 & 233 & 223 \\
\hline 4.624 & 0.24 & 1.60 & 1.81 & 1.91 & 312 & 232 & 223 & 220 \\
\hline 4.749 & 0.25 & 1.55 & 1.67 & 1.98 & 317 & 237 & 232 & 220 \\
\hline 5.563 & 0.18 & 1.53 & 1.78 & 1.95 & 313 & 233 & 223 & 216 \\
\hline 5.662 & 0.29 & 1.48 & 1.73 & 1.89 & 310 & 239 & 228 & 221 \\
\hline 5.763 & 0.10 & 1.49 & 1.56 & 1.81 & 322 & 236 & 233 & 223 \\
\hline 5.858 & 0.11 & 1.46 & 1.53 & 1.80 & 317 & 235 & 233 & 221 \\
\hline 5.972 & 0.18 & 1.45 & 1.71 & 1.85 & 319 & 240 & 229 & 223 \\
\hline 6.091 & 0.10 & 1.44 & 1.51 & 1.79 & 318 & 237 & 234 & 222 \\
\hline 6.14 & 0.25 & 1.52 & 1.71 & 1.90 & 316 & 239 & 230 & 222 \\
\hline 6.199 & 0.18 & 1.46 & 1.59 & 1.80 & 310 & 235 & 229 & 221 \\
\hline 6.277 & 0.21 & 1.42 & 1.61 & 1.89 & 309 & 237 & 229 & 218 \\
\hline 6.417 & 0.19 & 1.46 & 1.65 & 1.79 & 313 & 237 & 229 & 223 \\
\hline 6.764 & 0.16 & 1.53 & 1.59 & 1.87 & 312 & 232 & 229 & 218 \\
\hline 7.04 & 0.17 & 1.55 & 1.72 & 1.86 & 321 & 237 & 229 & 224 \\
\hline 7.184 & 0.24 & 1.58 & 1.67 & 1.87 & 312 & 233 & 229 & 222 \\
\hline 7.333 & 0.15 & 1.51 & 1.66 & 1.83 & 319 & 236 & 229 & 222 \\
\hline 9.001 & 0.06 & 1.46 & 1.55 & 1.76 & 314 & 232 & 228 & 220 \\
\hline Min. deviation: & -0.28 & -0.16 & -0.25 & -0.21 & -16 & -6 & -7 & -5 \\
\hline Average values: & 0.34 & 1.58 & 1.76 & 1.97 & 312 & 238 & 230 & 221 \\
\hline Max. deviation: & 0.70 & 0.52 & 0.58 & 0.53 & 9 & 9 & 7 & 3 \\
\hline
\end{tabular}
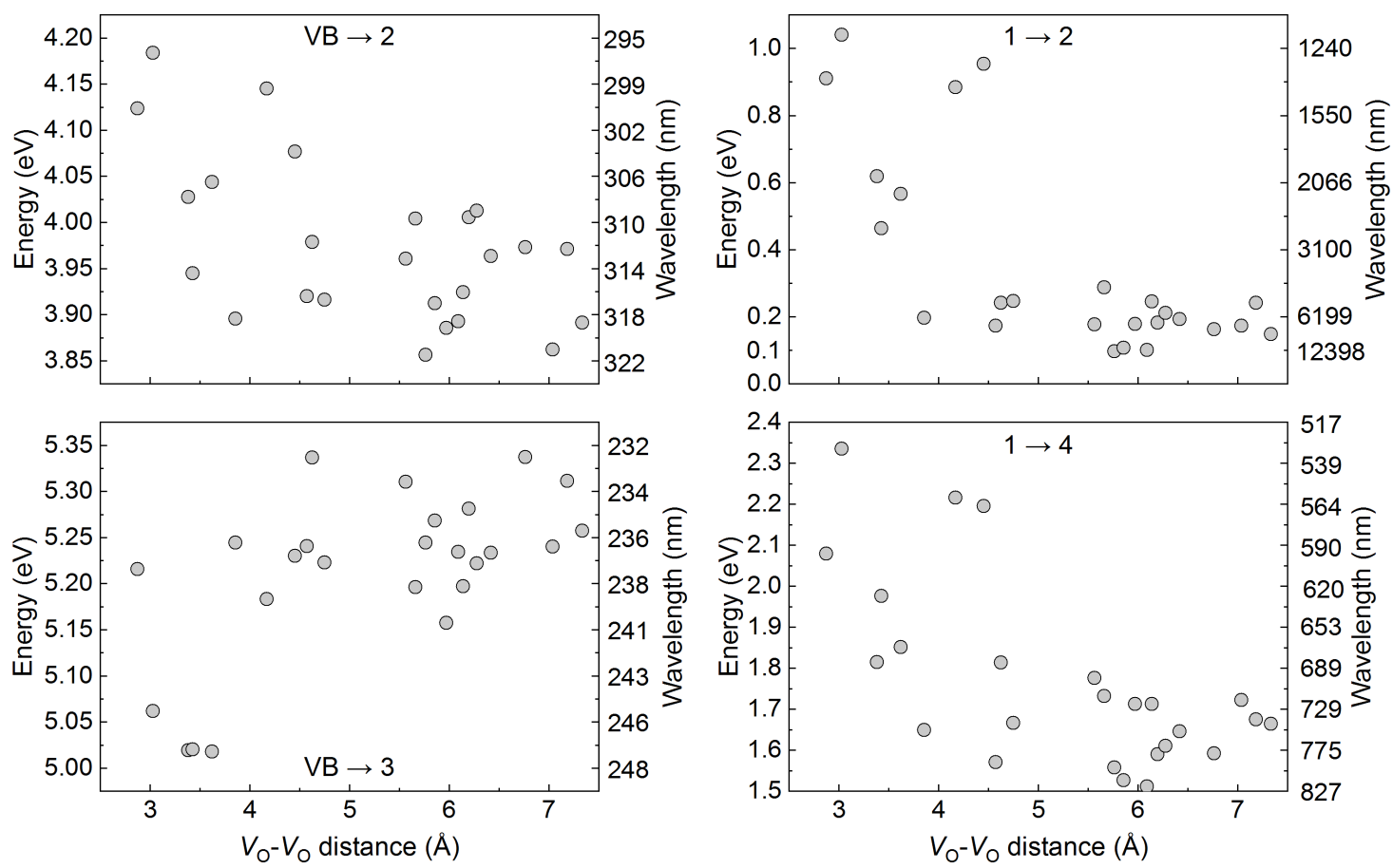

Fig. S5. Trends in energy differences between selected bands of $\mathrm{Lu}_{2} \mathrm{O}_{3}:\left(V_{\mathrm{O} 33}, V_{\mathrm{O} . . .}\right)^{\bullet \bullet} \mathrm{TDOS}$, and the corresponding wavelengths 
Table S8. Wavelengths of possible transitions in $\mathrm{Lu}_{2} \mathrm{O}_{3}: V_{\mathrm{O} 33}^{\bullet \bullet}, V_{\mathrm{O}}^{\bullet \bullet}$.

\begin{tabular}{cccccc}
\hline $\mathrm{R}_{V-V}, \AA$ & $\mathrm{CB} \rightarrow 1$ & $\mathrm{CB} \rightarrow 2$ & $\mathrm{CB} \rightarrow 3$ & $\mathrm{CB} \rightarrow 4$ & $\mathrm{CB} \rightarrow \mathrm{VB}$ \\
\hline 2.874 & 436 & 313 & 246 & 241 & 233 \\
3.026 & 443 & 323 & 268 & 240 & 232 \\
3.38 & 348 & 308 & 249 & 238 & 226 \\
3.428 & 358 & 320 & 258 & 231 & 228 \\
3.622 & 343 & 302 & 251 & 234 & 225 \\
3.857 & 333 & 322 & 243 & 239 & 226 \\
4.172 & 477 & 311 & 252 & 242 & 229 \\
4.452 & 423 & 305 & 256 & 240 & 233 \\
4.574 & 324 & 308 & 242 & 238 & 226 \\
4.624 & 316 & 299 & 238 & 231 & 228 \\
4.749 & 328 & 305 & 239 & 235 & 225 \\
5.563 & 323 & 306 & 241 & 237 & 227 \\
5.662 & 344 & 315 & 249 & 234 & 227 \\
5.763 & 318 & 310 & 239 & 224 & 219 \\
5.858 & 317 & 308 & 237 & 225 & 216 \\
5.972 & 332 & 316 & 249 & 234 & 223 \\
6.091 & 314 & 307 & 242 & 236 & 225 \\
6.14 & 324 & 302 & 246 & 234 & 224 \\
6.199 & 317 & 301 & 240 & 231 & 224 \\
6.277 & 317 & 298 & 241 & 232 & 221 \\
6.417 & 319 & 302 & 238 & 229 & 225 \\
6.764 & 319 & 304 & 235 & 223 & 216 \\
7.04 & 340 & 324 & 247 & 236 & 230 \\
7.184 & 330 & 309 & 238 & 233 & 226 \\
7.333 & 323 & 311 & 241 & 233 & 224 \\
9.001 & 308 & 302 & 234 & 231 & 222 \\
\hline Min. deviation: & -37 & -10 & -10 & -11 & -9 \\
Average values: & 345 & 309 & 245 & 234 & 225 \\
Max. deviation: & 132 & 15 & 24 & 8 & 8 \\
& & & & &
\end{tabular}
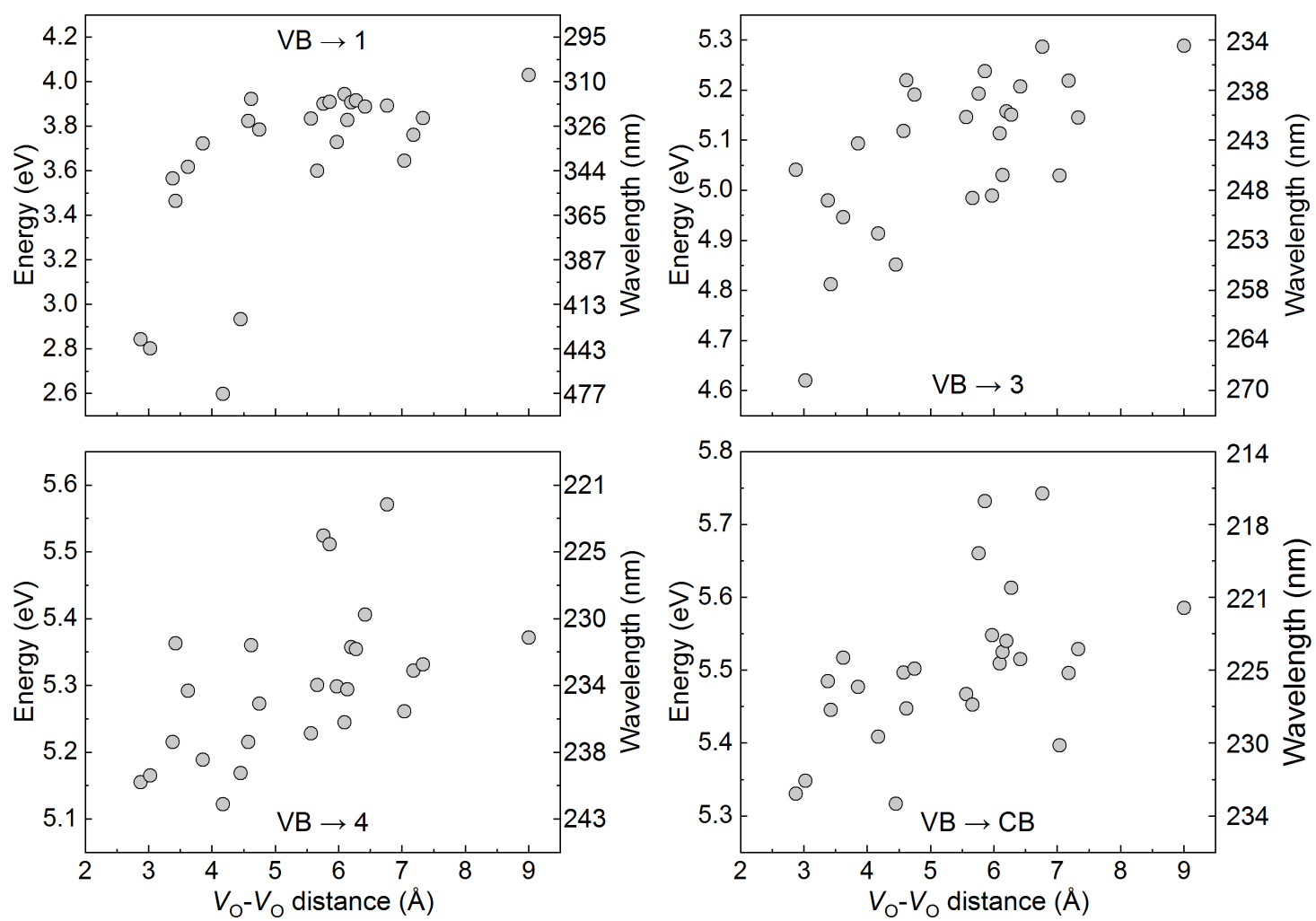

Fig. S6. Trends in energy differences between selected bands of $\mathrm{Lu}_{2} \mathrm{O}_{3}: V_{\mathrm{O} 33}^{\bullet \bullet}, V_{\mathrm{O}}^{\bullet \bullet}$. TDOS, and the corresponding wavelengths. 


\section{Frenkel pairs - total energy changes}

With the initial positions of $\mathrm{O}_{\mathrm{i}}$ at proximity of the $V_{\mathrm{O}}$, the $\mathrm{O}_{\mathrm{i}}$ have moved to the $V_{\mathrm{O}}$ site. In $\mathrm{Lu}_{2} \mathrm{O}_{3}: V_{\mathrm{O} 33}^{\bullet \bullet}, \mathrm{O}_{\mathrm{i} 01}^{\prime \prime}$ (Table S6), geometry optimization resulted in a transfer of $\mathrm{O}_{\mathrm{i}}$ from its initial position at fc. $0.375,0.375,0.375$ to fc. $0.3911,0.1551,0.380$. In $\mathrm{Lu}_{2} \mathrm{O}_{3}: V_{\mathrm{O} 33}^{\bullet \bullet}, \mathrm{O}_{\mathrm{i} 05}^{\prime \prime}, \mathrm{O}_{\mathrm{i}}$ have moved from fc. $0.625,0.125,0.375$ fc. $0.3942,0.1520,0.380$. The coordinates of the two oxygen atoms in the final geometries correspond approximately to the O33 site (Table S2, fc. 0.3913, $0.1524,0.380$ ) of the optimized pristine $\mathrm{c}-\mathrm{Lu}_{2} \mathrm{O}_{3}$.
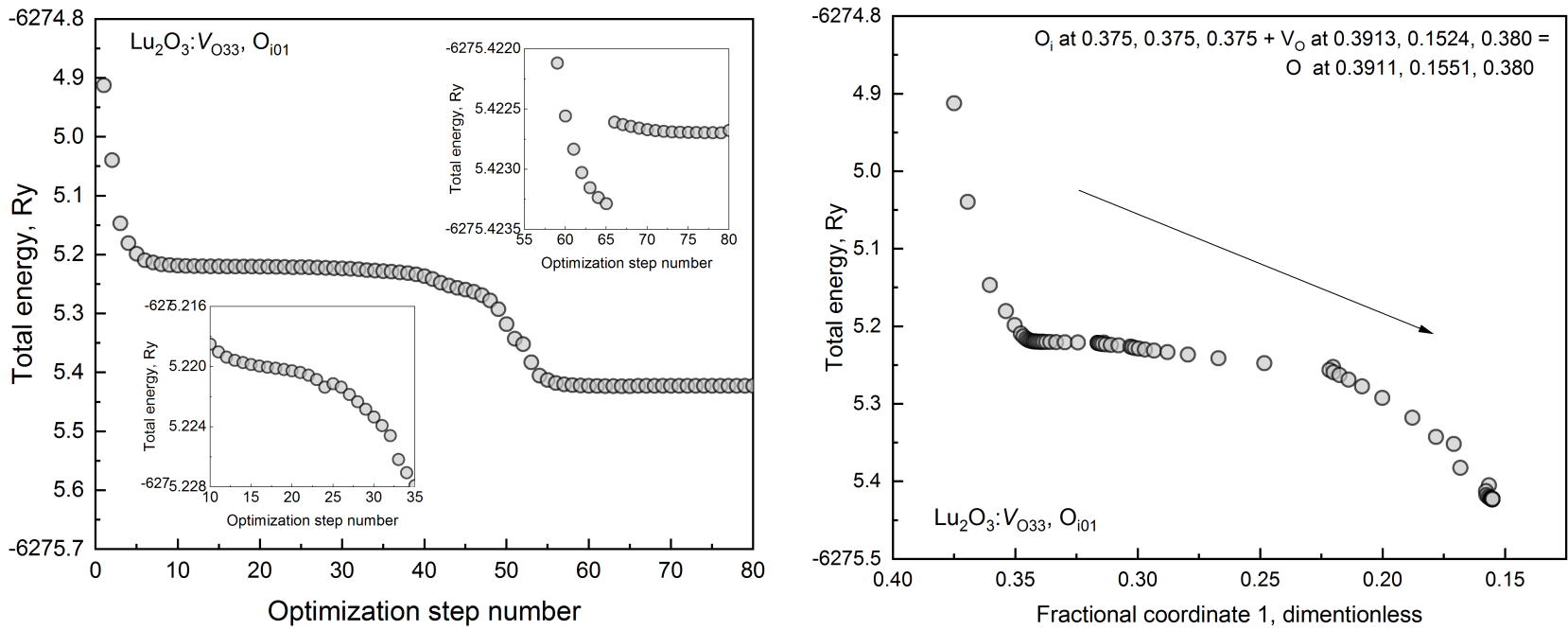

Fig. S7. Total energy changes during geometry optimization of $\mathrm{Lu}_{2} \mathrm{O}_{3}: V_{\mathrm{O} 33}^{\bullet \bullet}, \mathrm{O}_{\mathrm{i} 01}^{\prime \prime}$ as a function of optimization step (left) and fractional coordinate 2 changing from 0.375 to 0.1551 (right).

The insets show zoomed parts of the left plot. Discontinuity at steps 65-66 (left plot, upper inset) is caused by the calculation interruption and restart.
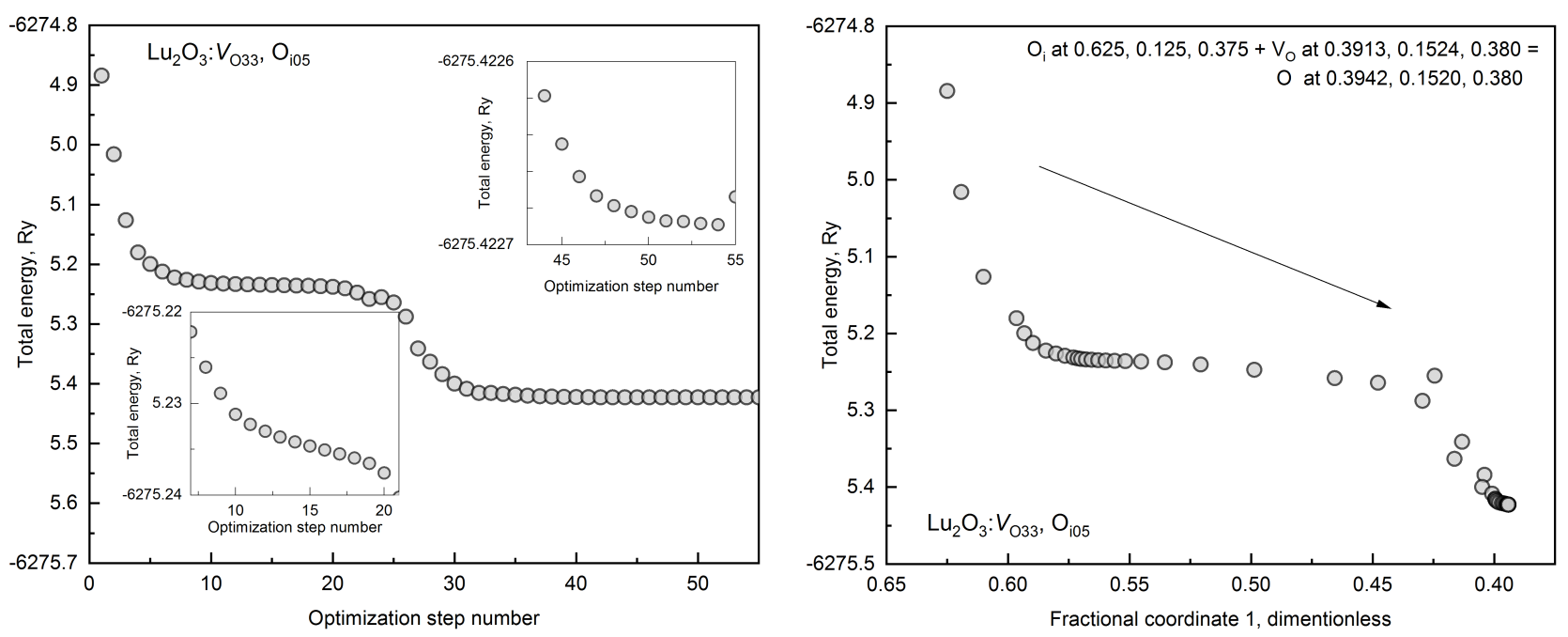

Fig. S8. Total energy changes during geometry optimization of $\mathrm{Lu}_{2} \mathrm{O}_{3}: V_{\mathrm{O} 33}^{\bullet \bullet}, \mathrm{O}_{\mathrm{i} 05}^{\prime \prime}$ as a function of optimization step (left) and fractional coordinate 1 changing from 0.625 to 0.3942 (right).

The insets show zoomed parts of the left plot. 


\section{Frenkel pairs $-O_{i}$ coordinates and trap depths}

Table S9. Fractional coordinates of $\mathrm{O}_{\mathrm{i}}$ for the Frenkel defects

\begin{tabular}{ccccc}
\hline Structure number & fc1 & fc2 & fc3 & R $\left(V_{\text {озз }}-\mathrm{O}_{\mathrm{i}}\right), \AA$ \\
\hline 01 & 0.375 & 0.375 & 0.375 & 2.296 \\
02 & 0.875 & 0.875 & 0.875 & 7.667 \\
03 & 0.625 & 0.625 & 0.625 & 5.979 \\
04 & 0.125 & 0.125 & 0.125 & 3.802 \\
05 & 0.625 & 0.125 & 0.375 & 2.420 \\
06 & 0.125 & 0.625 & 0.875 & 7.552 \\
07 & 0.375 & 0.875 & 0.625 & 3.809 \\
08 & 0.875 & 0.375 & 0.125 & 6.071 \\
09 & 0.375 & 0.625 & 0.125 & 5.525 \\
10 & 0.875 & 0.125 & 0.625 & 5.583 \\
11 & 0.625 & 0.375 & 0.875 & 6.077 \\
12 & 0.125 & 0.875 & 0.375 & 3.954 \\
13 & 0.625 & 0.875 & 0.125 & 4.559 \\
14 & 0.125 & 0.375 & 0.625 & 4.369 \\
15 & 0.375 & 0.125 & 0.875 & 5.101 \\
16 & 0.875 & 0.625 & 0.375 & 6.955 \\
\hline
\end{tabular}

Table S10. Band gap and trap depths (eV) of the structures with Frenkel defect

\begin{tabular}{ccccccccc}
\hline Structure number & $\mathrm{R}\left(V_{\text {озз- }} \mathrm{O}\right), \AA$ & Band gap & $\mathrm{CB}-\mathrm{O}_{\mathrm{i} 1}$ & $\mathrm{CB}-\mathrm{O}_{\mathrm{i} 2}$ & $\mathrm{CB}_{\mathrm{i}} \mathrm{O}_{\mathrm{i} 3}$ & $V_{\mathrm{O}-\mathrm{CB}}$ & $V_{\mathrm{O}}-V_{\mathrm{O}}^{*}$ & $V_{\mathrm{O}}^{*} \mathrm{CB}$ \\
\hline 02 & 7.667 & 5.42 & 0.265 & 0.467 & 0.543 & 1.50 & 1.21 & 0.29 \\
03 & 5.979 & 5.46 & 0.222 & 0.395 & 0.492 & 1.50 & 1.19 & 0.31 \\
04 & 3.802 & 5.36 & 0.041 & 0.230 & 0.305 & 1.35 & 1.18 & 0.16 \\
06 & 7.552 & 5.45 & 0.249 & 0.481 & 0.551 & 1.47 & 1.21 & 0.26 \\
07 & 3.809 & 5.47 & 0.226 & 0.349 & 0.437 & 1.45 & 1.14 & 0.31 \\
08 & 6.071 & 5.47 & 0.240 & 0.477 & 0.559 & 1.63 & 1.31 & 0.32 \\
09 & 5.525 & 5.52 & 0.226 & 0.463 & 0.540 & 1.55 & 1.39 & 0.17 \\
10 & 5.583 & 5.52 & 0.307 & 0.516 & 0.594 & 1.62 & 1.28 & 0.34 \\
11 & 6.077 & 5.46 & 0.254 & 0.479 & 0.562 & 1.56 & 1.26 & 0.30 \\
12 & 3.954 & 5.47 & 0.212 & 0.402 & 0.537 & 1.37 & 1.15 & 0.22 \\
13 & 4.559 & 5.65 & 0.221 & 0.369 & 0.455 & 1.40 & 1.11 & 0.29 \\
14 & 4.369 & 5.48 & 0.204 & 0.449 & 0.511 & 1.51 & 1.22 & 0.29 \\
15 & 5.101 & 5.53 & 0.058 & 0.389 & 0.533 & 1.31 & 1.06 & 0.25 \\
16 & 6.955 & 5.43 & 0.251 & 0.483 & 0.484 & 1.34 & 1.17 & 0.17 \\
\hline
\end{tabular}

\section{Fig. 14 color mapping}

The Vis colors in Fig. 9 of the main text were mapped via conversion of transition energy into wavelength, and then comparing the wavelength to CIE 1964 standard observer, using sRGB matrix + gamma correction. The implementation was based on the StackOverflow user amartynov's answer in the following thread: https://stackoverflow.com/questions/1472514/convert-light-frequency-to-rgb 